\title{
A Modified Social Spider Optimization for Economic Dispatch with Valve-Point Effects
}

\author{
Wenqiang Yang, ${ }^{1,2}$ Tingli Cheng, ${ }^{3}$ Yuanjun Guo, ${ }^{4}$ Zhile Yang $\mathbb{D},{ }^{4}$ and Wei Feng $\mathbb{C}^{4}$ \\ ${ }^{1}$ Postdoctoral Research Base, Henan Institute of Science and Technology, Xinxiang 453003, Henan, China \\ ${ }^{2}$ Postdoctoral Station, Henan University of Science and Technology, Luoyang 471000, Henan, China \\ ${ }^{3}$ Hefei University of Technology, Hefei 230009, Anhui, China \\ ${ }^{4}$ Shenzhen Institutes of Advanced Technology, Chinese Academy of Sciences, Shenzhen 518055, Guangdong, China \\ Correspondence should be addressed to Zhile Yang; zl.yang@siat.ac.cn and Wei Feng; wei.feng@siat.ac.cn
}

Received 16 July 2020; Revised 9 September 2020; Accepted 26 September 2020; Published 20 October 2020

Academic Editor: Qiang Chen

Copyright (c) 2020 Wenqiang Yang et al. This is an open access article distributed under the Creative Commons Attribution License, which permits unrestricted use, distribution, and reproduction in any medium, provided the original work is properly cited.

\begin{abstract}
Economic dispatch (ED) aims to allocate the generation of units to minimize the total production cost. This dispatch is generally formulated with nonsmooth and nonconvex cost function due to valve-point effects and various constraints, where the conventional methods are inapplicable. An improved social spider optimization algorithm, namely, ISSO, is proposed in this paper to solve the ED problem with valve-point effects. That is, dynamic updating mechanism of the subpopulations, Gaussian mating radius, and multimating strategy are introduced into the ISSO. These mechanisms facilitate a compromise between the global exploration and local exploitation of the search process. Numerical experiments are conducted on benchmark functions and different scale generation units commonly considered in the literature to validate the feasibility of the proposed ISSO. Computational results are analyzed in terms of solution quality by the statistical method, which shows the superiority of the ISSO algorithm in comparison with the state-of-the-art algorithms.
\end{abstract}

\section{Introduction}

Economic dispatch (ED) is one of the important issues in the power system. The objective of ED is to save the power generation cost while satisfying all kinds of operational constraints [1-4]. However, ED has nonsmooth, nonlinear, nonconvex, and nondifferentiable characteristics when valve-point effects of generation units are considered. Classical mathematical optimal approaches, such as Lagrangian relaxation [5], linear programming [6], branch and bound [7], and quadratic programming [8], are infeasible in solving ED problems due to the aforementioned characteristics. With the development of intelligent optimization theory and computer technology, metaheuristics based on natural evolution, as a novel simulated evolutionary computation technology, have shown their outstanding performance in solving complex optimization problems due to the absence of special requirements for the objective function. This condition guarantees the search for an effective solution within a time limit by a large probability. In recent decades, metaheuristic algorithms, which have got fast development [9-12], are utilized to solve ED problems with valve-point effects, such as genetic algorithm (GA) $[13,14]$, particle swarm optimization (PSO) [15-19], grey wolf optimization (GWO) [20], simulated annealing (SA) [21], bat algorithm (BA) [22], biogeography-based optimization (BBO) [23], differential evolution (DE) [24], whale optimization algorithm (WOA) [25], teaching-learningbased optimization (TLBO) [26], and cuckoo search algorithm (CSA) [27, 28].

Although the progress of the above methods has been made for the applicability to ED problems, the complexity of the ED problem reveals the necessity for the development of efficient algorithms to precisely locate the optimal solution. Within this context, the contribution of this paper is to develop a novel method for solving the ED problem, aiming 
to provide a workable solution for ED problems. Considering the intelligent behavior of a gregarious colony of spiders, a novel metaheuristic optimization approach called social spider optimization (SSO) is proposed by Cuevas et al. [29], which is simple, easy to realize, and adaptable to a wide range of optimization fields [30-33]. However, SSO has the following drawbacks during the evolution process: one is that interactive learning within female subpopulation or male subpopulation makes subpopulation similar; another one is that the fixed mating radius reduces convergence speed; and the last one is that single mating operator decreases the possibility of introducing good genes into offspring. All these drawbacks may lower the diversity of the population and eventually lead to premature convergence. Hence, a variant of SSO, namely, ISSO, including dynamic updating mechanism of the subpopulations, Gaussian mating radius, and multimating strategy, is proposed to solve ED problems with valve-point effects and enhance the performance of the conventional SSO. Furthermore, updating mechanism of the subpopulations and Gaussian mating is different from other references, which is illustrated in detail in Section 4.

The remainder of this paper is organized as follows. Section 2 presents the formulation of the ED problem with valve-point effects. Section 3 describes the conventional SSO, followed by Section 4 where the proposed ISSO for solving the ED problem is proposed in detail. Section 5 evaluates comprehensively the performance of the proposed method on benchmark problems with the comparative study. Finally, the paper ends with conclusions and further research works.

\section{Problem Statement}

2.1. Objective Function. The ED is a complicated optimal decision problem in power systems that allocates the generation of units to minimize the total fuel cost under a given load demand. Additionally, the ED problem in this work must satisfy operation constraints, which can be generally defined by a polynomial function as follows:

$$
\min F=\sum_{i=1}^{n} F_{i}\left(P_{i}\right)
$$

where $F, P_{i}$, and $n$ are the total fuel cost for all generation units, the power output of the $i$ th generation unit, and the number of generation units, respectively. $F_{i}\left(P_{i}\right)$ is the fuel cost of the $i$ th generation unit and usually expressed as a quadratic function:

$$
F_{i}\left(P_{i}\right)=a_{i} P_{i}^{2}+b_{i} P_{i}+c_{i} .
$$

The valve-point effect is a problem that generally cannot be ignored for thermal power generation units. Thus, the ED problem becomes nonsmooth and nonconvex. The valvepoint effect, which is generally equivalent to a sinusoidal term, must be involved in modeling the ED problem to solve the practical ED problem accurately. Therefore, the fuel cost function considering the valve-point effects of the generating units is given by

$$
F_{i}\left(P_{i}\right)=a_{i} P_{i}^{2}+b_{i} P_{i}+c_{i}+\left|e_{i} \times \sin \left(f_{i} \times\left(P_{i}^{\min }-P_{i}\right)\right)\right|,
$$

where $a_{i}, b_{i}, c_{i}, e_{i}$, and $f_{i}$ are the generation cost coefficients of the $i$ th generation unit and $P_{i}^{\min }$ is the minimum power output.

2.2. Constraints. The main constraints related to the ED problem are as follows:

(i) Power balance constraints

$$
\sum_{i=1}^{n} P_{i}=P_{D}+P_{L}
$$

where $P_{D}$ and $P_{L}$ are the total power demand and the total transmission loss, respectively. While power network is concentrated, thus $P_{L}$ is ignored.

(ii) Power output limits

$$
P_{i}^{\min } \leq P_{i} \leq P_{i}^{\max },
$$

where $P_{i}^{\min }$ and $P_{i}^{\max }$ are the minimum and the maximum power outputs of the $i$ th generation unit, respectively.

\section{Conventional SSO}

A new swarm intelligence algorithm called SSO was proposed by Cuevas et al. [29] considering the predation behavior of the spiders. Herein, the position of spiders corresponds to the solution of optimization problem. Meanwhile, the spider web, which is associated with the search space of optimization problems, is employed to facilitate interaction among spiders through the vibrations of spiders. Furthermore, the mating behavior of the female and male spiders, which is considered to be coevolution, serves to obtain the optimal or quasioptimal of the objective function. The basic principles of conventional SSO can be summarized as follows.

3.1. Initialization of the Population. The most prominent characteristic that differentiates the spiders from all other species is highly female-biased. The number of females $N_{f}$ accounts for $65 \%-90 \%$ of the entire population $N$, which is defined as follows:

$$
N_{f}=\text { floor }[(0.9-\operatorname{rand} 1 \times 0.25) \times N],
$$

where rand $1 \in[0,1]$ is a random number. In the spider population comprising female and male spiders, the number of males $N_{m}$ can be calculated as follows:

$$
N_{m}=N-N_{f}
$$

The population of $N$ spiders is randomly initialized, for any spider $i \in\{1,2, \ldots, N\}$, and its position is presented by a vector as the parameter values to be optimized, which represents one solution of the optimization problem. 
Herein, female and male spiders can be initialized according to the following equation:

$$
\left\{\begin{array}{l}
F_{i j}=p_{j \text { min }}+\operatorname{rand} 2 \times\left(p_{j \text { max }}-p_{j \text { min }}\right), \\
M_{k j}=p_{j \text { min }}+\operatorname{rand} 3 \times\left(p_{j \max }-p_{j \text { min }}\right),
\end{array}\right.
$$

where rand 2 and rand 3 , which are uniformly distributed between 0 and 1 , are random numbers. $F_{i j}$ and $M_{k j}$ are the $j$ th dimension of the position of the $i$ th female spider and the $j$ th dimension of the $k$ th male spider, respectively. $p_{j \min }$ and $p_{j \max }$ correspond to the minimum and maximum values of the $j$ th dimension of the spider.
3.2. Female Cooperative Operator. The positions of female spiders are updated in accordance with the vibration on the communal web generated by the superior spiders. Such vibration denotes an attraction or repulsion over other spiders, which is determined by probability factor PF. That is, for a random number $r_{m} \in[0,1]$, if $r_{m}$ is smaller than PF, then an attraction operation is performed; otherwise, a repulsion operation is executed. Considering the minimization problem in this paper, the mathematical models of such cooperative operator are defined as follows:

$$
\begin{aligned}
F_{i}^{t+1} & = \begin{cases}F_{i}^{t}+\alpha V i b_{c i}\left(S_{c}-F_{i}^{t}\right)+\beta V i b_{b i}\left(S_{b}-F_{i}^{t}\right)+\delta(\operatorname{rand} 4-0.5), & r_{m} \leq \mathrm{PF}, \\
F_{i}^{t}-\alpha V i b_{c i}\left(S_{c}-F_{i}^{t}\right)-\beta V i b_{b i}\left(S_{b}-F_{i}^{t}\right)+\delta(\operatorname{rand} 5-0.5), & r_{m}>\mathrm{PF},\end{cases} \\
V i b_{i j} & =w_{j} * e^{-d_{i j}^{2}}, \\
w_{i} & =\frac{J\left(S_{i}\right)-\text { worst }_{S}}{\text { best }_{S}-\text { worst }_{S},} \\
\text { best }_{S} & =\min \left\{J\left(S_{k}\right)\right\}, \\
\text { worst }_{S} & =\max \left\{J\left(S_{k}, \ldots, N\right\},\right.
\end{aligned}
$$

where $F_{i}^{t}$ indicates the position of the $i$ th female spider in $t$ th iteration. $\alpha, \beta$, and $\delta$ are random numbers between 0 and 1 . The weight $w_{i}$ represents the solution quality of the $i$ th spider. $d_{i j}$ is the Euclidian distance between the spiders $i$ and j. $S_{c}$ and $S_{b}$ denote the closest members, which have a high weight to the female spider $i$ and the best spider in the population $S$, respectively. $V i b_{i j}$ is the vibration perceived by the $i$ th spider generated by the $j$ th spider. In the end, $J\left(S_{i}\right)$ is the fitness value of the $i$ th spider position with respect to the objective function.
3.3. Male Cooperative Operator. From a biological viewpoint, male spiders $M$ comprise dominant and nondominant individuals. Herein, dominant individuals are those that have better weight than the median male spider. All the male spiders are sorted in descending order of weights in advance to obtain the median male spider $N_{f}+m$, and the individual located in the middle is considered the median male member. By contrast, other male spiders are nondominant individuals. The dominant individuals can attract the closest female spider. However, nondominant individuals gather around the median male member. Thus, the position of the male spider $i$ can be updated using the following equation:

$$
M_{i}^{t+1}= \begin{cases}M_{i}^{t}+\alpha V i b_{f i}\left(S_{f}-M_{i}^{t}\right)+\delta(\operatorname{rand} 6-0.5), & \omega_{N_{f}+i} \geq \omega_{N_{f}+m}, \\ M_{i}^{t}+\alpha\left(\frac{\sum_{h=1}^{N_{m}} M_{h}^{t} \omega_{N_{f}+h}}{\sum_{h=1}^{N_{m}} \omega_{N_{f}+h}}-M_{i}^{t}\right), & \text { else, }\end{cases}
$$

where $S_{f}$ and $\sum_{h=1}^{N_{m}} M_{h}^{t} \omega_{N_{f}+h} / \sum_{h=1}^{N_{m}} \omega_{N_{f}+h}$ represent the female spider closest to male spider $i$ and the mean weight of all the male spiders, respectively.
3.4. Mating Operator. Any dominant male spider $M_{G}$ can possibly mate with the female spiders. Under such circumstances, when the female spiders within a mating radius 
$r$, which is calculated by equation (14), form the set of mating members $T_{G}$ (that is, $T_{G}$ must not be empty), then the mating operation can be performed between dominant males and $T_{G}$. Furthermore, the roulette method is adopted to generate offspring. This method guarantees that the bigger the weight of the spider $i \in T_{G}$ is, the more chance it will have to reproduce. Therefore, the probability $P S_{i}$ by which each spider $i \in T_{G}$ is selected to mate is described by equation (15):

$$
\begin{aligned}
r & =\sum_{j=1}^{n} \frac{\left(p_{j \max }-p_{j \min }\right)}{2 n}, \\
P S_{i} & =\frac{\omega_{i}}{\sum_{k \in T_{G} \cup M_{G} \omega_{k}}}, \quad i \in T_{G} \cup M_{G},
\end{aligned}
$$

where $n$ is the dimension size of the problem. After mating, the acceptance of new spider $S_{\text {new }}$ depends on its weight. If the weight $\omega_{\text {new }}$ of the new spider $S_{\text {new }}$ is larger than the weight $\omega_{o}$ of the worst spider $S_{o}$ of the entire spider population, then the worst spider will be replaced with the new one; otherwise, the new spider $S_{\text {new }}$ is discarded. Once the replacement has occurred, the new spider $S_{\text {new }}$ will have the same gender as the replaced one to maintain the population. The flowchart of the SSO algorithm is described in Figure 1.

\section{Proposed ISSO}

4.1. Subpopulation Dynamically Updating Strategy. According to the principle of SSO, cooperative operators, which are all used within the female and male spiders, contribute to learning from each other within subpopulations. Hence, the difference among individuals gradually decreases with evolution. This reduction lowers the convergence rate and even results in prematurely getting stuck in the local optimal. Updating subpopulations is essential to overcome such shortcomings and mitigate their negative influence. Subpopulation strategy, which equates to multipopulation to some extent, has been applied in many swarm intelligence evolutionary algorithms before. However, the updating strategy of subpopulations, which is based on the subpopulation similarity, has been rarely considered during the evolution process in [34-36]. Nevertheless, improved subpopulation diversity for the entire population can guarantee that the search spaces from each subpopulation have relatively small overlaps. Thus, subpopulation separately evolves in each region, which is comparatively independent of the others. As previously mentioned, it can further help SSO to search toward the global optimal solution, and the global searching capability is considerably strengthened. To this end, a subpopulation dynamically updating strategy, which is utilized to improve the optimization capability of the subpopulation, is proposed in this paper. In this strategy, an index $\phi$, which can reflect the diversity of the population, is defined as equation (17) and employed to determine how each subpopulation fulfills the update requirement. This index mainly realizes information exchange between subpopulations through an evolution operator called migration operator. Specifically, the migration operator indicates that a better spider from the best subpopulation diversity, which is furthest from the best spider from the worst subpopulation diversity, migrates and replaces the worst spider. Considering the emigrating operation, the subpopulation is where a new spider is randomly generated to maintain the size of the subpopulation constant. For clarity, the subpopulation dynamically updating strategy during the evolution process is illustrated in Figure 2 .

Definition 1. Suppose the $i$ th spider $S_{i}$ is the best solution of the subpopulation thus far. The Euclidian distance $d_{i j}$ between $S_{i}$ and $S_{j}$ is adopted and mathematically modeled as shown below to measure the distance from $S_{i}$ to other spider $S_{j}$ :

$$
d_{i j}=\sqrt{\sum_{k=1}^{n}\left(s_{i k}-s_{j k}\right)^{2}}, \quad i \in\{1,2, \ldots, n\} \backslash\{j\},
$$

where $s_{i k}$ and $s_{j k}$ are the $k$ th decision variables in the solutions $S_{i}$ and $S_{j}$, respectively, and $n$ is the number of solution dimensions.

Definition 2. Designing an index of population diversity measuring the quality of the structure of subpopulation is necessary to address the challenging issue of effectively updating the operation of subpopulations. This index $\varphi$, which includes total distance $D_{s}$ and total fitness $F_{\text {total }}$, can be formulated as follows:

$$
\begin{aligned}
\varphi & =\varepsilon_{1} \times D_{s}+\varepsilon_{2} \times F_{\text {total }}, \\
D_{s} & =\sum_{i \in\left\{1,2, \ldots, N_{s}\right\} \backslash\{j\}} d_{i j}, \\
F_{\text {total }} & =\sum_{i=1}^{N_{s}} J\left(S_{i}\right),
\end{aligned}
$$

where $\varepsilon_{1}$ and $\varepsilon_{2}$ are the weight coefficients of $D_{s}$ and $F_{\text {total }}$, respectively, which represent the importance of $D_{s}$ and $F_{\text {total }}$ to the population diversity index $\phi$. A large $D_{s}$ indicates a wide solution space. That is, $D_{s}$ provides a remarkable contribution to the population diversity compared with $F_{\text {total }}$. Based on the above consideration, $\varepsilon_{1}$ and $\varepsilon_{2}$ are set to 2 and 1 , respectively. Moreover, the detailed description of the subpopulation dynamically updating strategy is presented in Algorithm 1.

4.2. Gaussian Mating Radius. To the best of our knowledge, the spiders get close to one another with evolution generations. However, the evolution process of SSO shows that the mating radius always remains unchanged, leading to more female spiders within the mating radius. Thus, the quantity of the worse female spiders, which are mated with the dominant male spiders, gradually increases. This condition means that the fixed mating radius will result in the poor quality of mating. That is to say, SSO will spend some time searching for the optimal 


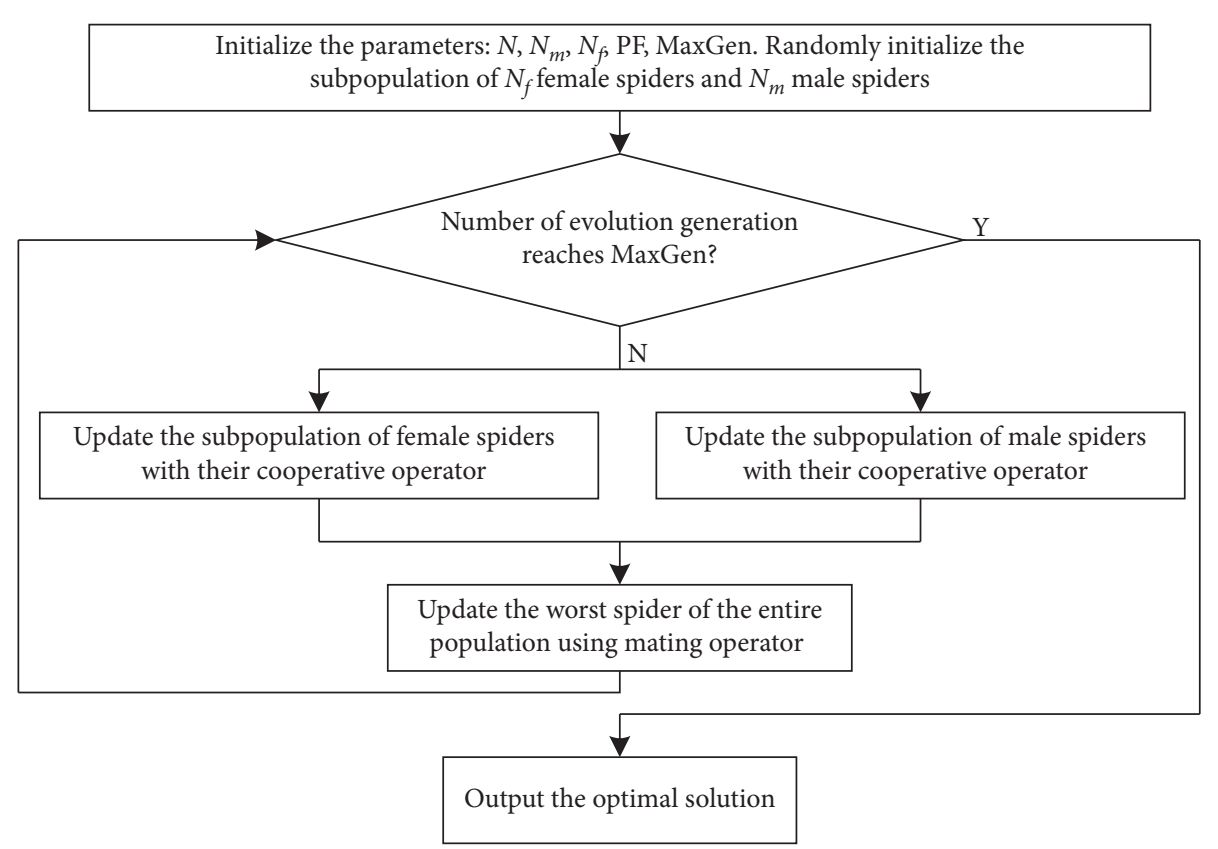

FIGURE 1: Flowchart of the original SSO algorithm.

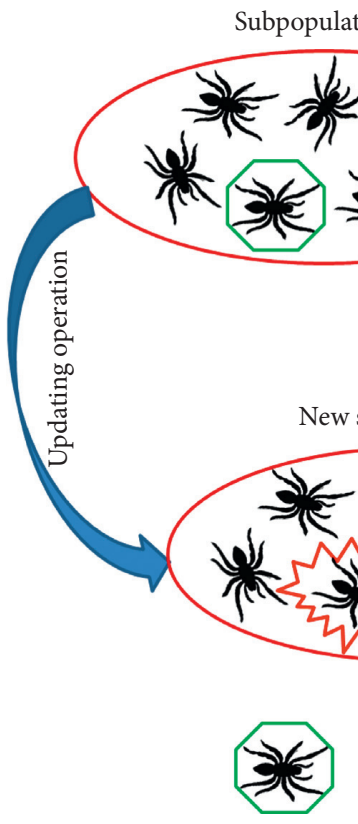

Worst spider

Figure 2: Subpopulation dynamically updating operation.

solution meaninglessly, making SSO less efficient and competitive. Introducing the adaptive mating radius is necessary to overcome such defects; that is, a large mating radius of the previous generation facilitates global optimization, while a small mating radius of the later generation improves the local optimal capability. In view of this, the oscillation decay strategy that combines negative exponential and Gaussian functions is proposed to guide the change in the mating radius. This strategy is comprehensively described as follows. The large mating radius, which corresponds to the large solution space, can rapidly guide the ISSO to approach the optimal or suboptimal solution in the previous period of search. Instead, ISSO will exploit the optimum by mating with high-quality female spiders in the later evolution stage. Furthermore, the overall mating radius decreases despite such oscillation. Owing to such characteristics, ISSO can provide a good balance between the exploration and the exploitation capabilities. Therefore, equation (14) can be modified by the following expression: 
(1) for each subpopulation $S P_{i}$ belonging to male subpopulation or female subpopulation

(2) for each individual $S_{i}$ in $S P_{i}$

(3) Calculate $J\left(S_{i}\right)$ for each $S_{i} \in S P_{i}$ according to the opposite of equation (1).

(4) Calculate $d_{i j}$ for each $S_{i}\left(S_{i}, S_{j} \in S P_{i}\right)$ according to the opposite of equation (1), where $S_{j}$ is the best individual.

(5) end for $S_{i}$

(6) Calculate $\phi_{i}$ for $S P_{i}$ according to equation (17).

(7) end for $S P_{i}$

(8) Determine the updated subpopulation denoted as $S P_{u}$ based on the small $\phi_{i}$. Another one denoted as $S P_{d}$. Then, all the spiders in $S P_{d}$ in descending order of the fitness and the top $20 \%$ of spiders are selected to form a set of candidate migrator $\Omega$.

(9) for each individual $S_{i}$ in $\Omega$

(10) Calculate $d_{i j}$ for each $S_{i}\left(S_{j} \in S P_{u}\right)$ according to the opposite of equation (1), where $S_{j}$ is the best individual.

(11) end for $S_{i}$

(12) Select the spider $S_{i} \in \Omega$ with the largest $d_{i j}$, which is used to replace the spider with the worst fitness in $S P_{u}$. Meanwhile, $S_{i}$ is replaced by new spider randomly generated in $S P_{d}$.

Algorithm 1: Pseudocode of the subpopulation dynamically updating strategy.

$$
r=\left[\sum_{j=1}^{n} \frac{\left(p_{j \max }-p_{j \min }\right)}{2 n}\right] \cdot e^{-(t / \mathrm{MaxGen})} \cdot N(\mu, \sigma),
$$

where $N(\mu, \sigma)$ is a random number of Gaussian distribution in $[0,1]$, and the mean and standard deviation are 0.5 and 0.16 , respectively. $t$ is the $t$ th generation of the evolution process, and MaxGen is the maximal number of generations.

4.3. Multimating Operator. The mating operator of SSO is an effective tool for ensuring high-quality offspring. Nevertheless, the genes from the dominant male spiders, which have not been fully inherited by the children, inevitably affect the solution quality of the next generation. Hence, modifications of original mating technology will be necessary to pass good genes from generation to generation. This technology can guide evolution toward promising areas. Based on the aforementioned analysis, the multimating strategy, which takes full advantage of the neighborhoods of parents, is presented to accelerate the solving process. Suppose two spiders $S_{m}=\left[s_{1 m}, s_{2 m}, \ldots, s_{n m}\right]$ and $S_{f}=\left[s_{1 f}, s_{2 f}, \ldots, s_{n f}\right]$ are two mating partners in the $t$ th generation. The improved multimating operator described above can be depicted as follows:

(a) Weighted mating operator

$$
s_{i m}^{\text {new }}=\operatorname{rand} 7 \cdot s_{i m}+[1-\operatorname{rand} 7] \cdot s_{i f} \text {. }
$$

(b) Average mating operator

$$
s_{i m}^{\text {new }}=\frac{s_{i m}+s_{i f}}{2} .
$$

(c) Extreme mating operator

$$
s_{i m}^{\text {new }}=\lfloor\operatorname{rand} 8\rfloor \cdot s_{i m}+(1-\lfloor\operatorname{rand} 8\rfloor) \cdot s_{i f} \text {. }
$$

(d) Bound mating operator

$$
s_{\text {im }}^{\text {new }}=\lfloor\operatorname{rand} 9\rfloor \cdot p_{\text {imin }}+(1-\lfloor\text { rand9 }\rfloor) \cdot p_{\text {imax }} \cdot
$$

In equations (21)-(24), rand7, rand8, and rand9 are all random numbers in the range $[0,1]$. The symbol $\lfloor\cdot\rfloor$ is an operator through which fractions are rounded down. $p_{i \text { min }}$ and $p_{i \max }$ are the upper and lower bounds of the $i$ th dimension of the spider, respectively. Notably, the proposed multimating operators provide remarkable efforts to compromise with the local and the global explorations by utilizing the neighborhood of the two mating spiders and the bound of the solution space. Thus, the chance of producing good solutions is significantly increased. The proposed mating approach can help overcome the limitations introduced by the origin mating operator. Considering the efficiency of the ISSO, one of the four proposed mating operators is randomly selected during mating operation. After the new offspring individual is produced, the new child is compared with the worst individual of the entire population. If this offspring is better in terms of fitness, then this offspring will replace the worst individual; otherwise, this offspring is discarded.

The pseudocode is summarized in Algorithm 2 to understand the principle of the proposed ISSO.

\section{Simulation Results}

In this section, the performance of the ISSO is tested by comparison with other variants of SSO, such as SSO [29], NISSO [37], MSSO [38], and OBSSO [39]. Comparisons with some state-of-the-art algorithms, which are the variants of PSO (CLPSO) [40] and DE (JADE) [41], are also conducted. The above experiments are performed by conducting a set of well-known benchmark functions [42], where functions $f_{1}-f_{4}$ and $f_{5}-f_{8}$ are unimodal and multimodal functions, respectively, as listed in Table 1. The unimodal function is easily solved due to only one optimum. By contrast, the number of local minimum increases with the dimension of problems in multimodal function, thus 
(1) Initialize the parameters: $N, N_{f}, N_{m}$, and PF.

(2) Initialize a subpopulation $S$ of $N_{f}$ female spiders and $N_{m}$ male spiders with random positions.

(3) Define the maximal number of iterations MaxGen and set $t=1$.

(4) while $(t<$ MaxGen)

(5) for $i=1: N$

(6) Evaluate the weight of each spider in the population $S$ using equation (11).

(7) end for $i$

(8) Record the best individual.

(9) for $j=1: N_{f}$

(10) Compute $V i b_{c i}$ and $V i b_{b i}$ by equation (10) and generate a random $r_{m} \in[0,1]$

(11) if $\left(r_{m} \leq \mathrm{PF}\right)$

(12) $F_{j}^{t+1}=F_{j}^{t}+\alpha V i b_{c j}\left(S_{c}-F_{j}^{t}\right)+\beta V i b_{b j}\left(S_{b}-F_{j}^{t}\right)+\delta(\operatorname{rand} 4-0.5)$

(13) else

(14) $F_{j}^{t+1}=F_{j}^{t}-\alpha V i b_{c j}\left(S_{c}-F_{j}^{t}\right)-\beta V i b_{b j}\left(S_{b}-F_{j}^{t}\right)+\delta(\operatorname{rand} 5-0.5)$

(15) end if

(16) end for $j$

(17) Calculate the weight $\omega_{N_{f}+m}$ of the median male spider from $M$.

(18) for $k=1: N_{m}$

(19) if $\left(\omega_{N_{f}+i} \geq \omega_{N_{f}+m}\right)$

(20) Determine the closest female $S_{f}$, and compute $V i b_{f i}$ by equation (10).

(21) $M_{k}^{t+1}=M_{k}^{t}+\alpha V i b_{f k}\left(S_{f}-M_{k}^{t}\right)+\delta(\operatorname{rand} 6-0.5)$

(22) else

(23) $M_{k}^{t+1}=M_{k}^{t}+\alpha\left(\left(\sum_{h=1}^{N_{m}} M_{h}^{t} \omega_{N_{f}+h} / \sum_{h=1}^{N_{m}} \omega_{N_{f}+h}\right)-M_{k}^{t}\right)$

(24) end if

(25) end for $k$

(26) Calculate the weights of the spiders, and the self-adaptive mating radius $r$ by equation (20).

(27) for $l=1: N_{m}$

(28) if $\left(M_{l}\right.$ is the dominant male spider)

(29) if $\left(T_{G}\right.$ is not empty)

(30) for $m=1: n$

(31) Select each dimension $s_{m f}$ of the female spider based on the roulette method.

(32) end for $m$

(33) All the dimensions selected, such as $s_{1 f}, s_{2 f}, \ldots$, and $s_{n f}$, form the female spider $S_{f}$.

(34) Randomly select one of the multimating operators among weighted, average, extreme, and bound mating operator.

(35) Generate the new spider $S_{\text {new }}$ by mating $M_{l}$ with $S_{f}$ based on the mating operator selected.

(36) if $\left(\omega_{\text {new }}>\omega_{o}\right)$

(37) $S_{o}=S_{\text {new }}$

(38) end if

(39) end if

(40) end if

(41) end for $l$

(42) Perform the subpopulation dynamically update strategy using Algorithm 1.

(43) $t=t+1$

(44) end while

(45) Output the best solution

Algorithm 2: Pseudocode of the proposed ISSO.

making it difficult to obtain optimal solutions. Therefore, considering the above analysis, the selected benchmark functions can effectively evaluate the performance of the algorithm in terms of escaping from the local optimum and convergence speed. Eventually, the ISSO is applied to the ED problem with valve-point effects.

All experiments in this study are conducted in a PC with Windows 10 system, 3.7 GHz Intel Core, 4 GB RAM, and MATLAB R2014b. For the parameters of each algorithm, the parameter $N$ follows the recommendation in [29] and is set to 50 , and the parameters of other algorithms are the same as those of the corresponding references, such as SSO in [29], NISSO in [37], MSSO in [38], OBSSO in [39], CLPSO in
[40], and JADE [41]. For fairness, each algorithm, which evaluates the function with $D$ dimension, terminates after reaching the maximum number of function evaluations (MaxFES). Herein, the benchmark functions with 50 dimensions are employed to examine the performance of the algorithms, of which MaxFES are set to 500,000. Simultaneously, each algorithm has 30 independent runs for each trial.

5.1. Sensitivity Analysis of the Probability factor PF. For the proposed ISSO, the female spider decides whether or not to approach the superior spider based on the probability factor PF 
TABLE 1: Details about benchmark functions.

\begin{tabular}{|c|c|c|c|}
\hline Name & Function & Search range & Min \\
\hline Sphere & $f_{1}(x)=\sum_{i=1}^{D} x_{i}^{2}$ & {$[-100,100]$} & 0 \\
\hline SumSquare & $f_{2}(x)=\sum_{i=1}^{D} i x_{i}^{2}$ & {$[-10,10]$} & 0 \\
\hline Schwefel 2.21 & $f_{3}(x)=\max \left\{\left|x_{i}\right|, 1 \leq x_{i} \leq D\right\}$ & {$[-100,100]$} & 0 \\
\hline Schwefel 2.22 & $f_{4}(x)=\sum_{i=1}^{D}\left|x_{i}\right|+\prod_{i=1}^{D}\left|x_{i}\right|$ & {$[-10,10]$} & 0 \\
\hline Rosenbrock & $f_{5}(x)=\sum_{i=1}^{D-1}\left\{100 \cdot\left(x_{i+1}-x_{i}^{2}\right)^{2}+\left(1-x_{i}\right)^{2}\right\}$ & {$[-5,10]$} & 0 \\
\hline Rastrigin & $f_{6}(x)=\sum_{i=1}^{D}\left(x_{i}^{2}-10 \cos \left(2 \pi x_{i}\right)+10\right)$ & {$[-5.12,5.12]$} & 0 \\
\hline Ackley & $\left.f_{7}(x)=-20 \exp (-0.2] \frac{1}{D} \sum_{i=1}^{D} x_{i}^{2}\right)$ & {$[-32,32]$} & 0 \\
\hline Levy & $\begin{array}{c}-\exp \left(\frac{1}{D} \sum_{i=1}^{D} \cos \left(2 \pi x_{i}\right)\right)+20+e \\
f_{8}(x)=\sum_{i=1}^{D-1}\left(x_{i}-1\right)^{2}\left[1+\sin ^{2}\left(3 \pi x_{i+1}\right)\right]+\sin ^{2}\left(3 \pi x_{1}\right) \\
+\left|x_{n}-1\right|\left[1+\sin ^{2}\left(3 \pi x_{n}\right)\right]\end{array}$ & {$[-10,10]$} & 0 \\
\hline
\end{tabular}

during the female cooperative stage. Thus, $\mathrm{PF}$ is an important parameter influencing the performance of the ISSO, and tuning the probability factor PF is crucial. Without loss of generality, three kinds of different test functions are selected to investigate the impact of the parameter PF. These test functions include Sphere, Schwefel2.22, and Ackley, with 50 dimensions as listed in Table 1 . The mean values of the 30 runs of the ISSO are presented in Table 2. As seen in Table 2, the variance of the PF has a powerful effect on the performance of the ISSO, and two out of three functions can obtain the best mean results with the PF 0.6. Thus, the PF should be set to 0.6 , where the performance of the ISSO is satisfactory.

5.2. Performance Evaluation. Table 3 shows the comparison results in terms of the minimum "Min," the mean "Mean," the standard deviation "Std" of the best-so-far solution, and the average computing time "At," where the best results are highlighted in boldface.

Table 3 shows that the proposed ISSO is better than any other SSO variants according to the statistical results. Meanwhile, CLPSO and JADE are also better behaved than SSO variants, such as SSO, NISSO, MSSO, and OBSSO. More importantly, the optimization performance of ISSO is superior to that of CLPSO and JADE for all benchmark functions, except the functions SumSquare, Rosenbrock, and Levy. Specifically, the small mean and standard deviation to different kinds of functions indicate the high solution precision and stability of ISSO. Furthermore, compared with other methods, ISSO significantly reduces the time of computing. Such improvements are related to these strategies, such as subpopulation dynamically updating strategy, Gaussian mating radius, and multimating operator, which are introduced into ISSO. These results fully demonstrate that the proposed ISSO is promising and competitive.
TABle 2: Comparisons of the results varying PF.

\begin{tabular}{lccc}
\hline PF & Sphere & Schwefel2.22 & Ackley \\
\hline 0.1 & $6.20 E-58$ & $3.16 E-26$ & $8.28 E-09$ \\
0.2 & $8.47 E-59$ & $4.82 E-28$ & $4.25 E-09$ \\
0.3 & $3.05 E-59$ & $9.24 E-29$ & $8.47 E-11$ \\
0.4 & $5.68 E-60$ & $6.11 E-29$ & $7.09 E-11$ \\
0.5 & $8.49 E-62$ & $5.92 E-30$ & $6.22 E-13$ \\
0.6 & $\mathbf{7 . 0 5 E}-\mathbf{6 2}$ & $5.75 E-31$ & $\mathbf{3 . 3 0 E}-\mathbf{1 4}$ \\
0.7 & $3.44 E-61$ & $\mathbf{4 . 8 4 E}-\mathbf{3 1}$ & $1.40 E-13$ \\
0.8 & $1.89 E-59$ & $2.15 E-30$ & $7.34 E-13$ \\
0.9 & $5.60 E-57$ & $4.38 E-28$ & $2.58 E-11$ \\
1 & $8.42 E-57$ & $6.87 E-28$ & $5.81 E-10$ \\
\hline
\end{tabular}

5.3. Application to ED Problem with Valve-Point Effects. Three cases from the reference [1], which are 3-unit, 13-unit, and 40-unit system ED problems with valve-point effects, are considered to verify the validity and feasibility of the proposed method ISSO for solving these problems. The detailed data on the three cases are provided in [1]. The results obtained by ISSO are compared with the aforementioned algorithms, including SSO [29], NISSO [37], MSSO [38], OBSSO [39], CLPSO [40], and JADE [41]. The maximum evolution generations of each case remain the same as [1] to realize a fair comparison. Thus, 3-unit, 13-unit, and 40-unit systems are, respectively, set to 50,800, and 1000, and other parameters are the same as those in Section 5.2. The comparison results are summarized as the minimum cost "Min," the mean cost "Mean," the maximum cost "Max," and the average computing time "At" in Tables 4-6, respectively. The best dispatch schemes corresponding to 3unit, 13-unit, and 40-unit systems, which are achieved by utilizing ISSO, are, respectively, listed in Tables 7-9. The mean values of 30 trials and the convergence performance curves are, respectively, illustrated in Figures 3 and 4 to 
TABle 3: Comparisons of the results among the algorithms with $D=50$.

\begin{tabular}{|c|c|c|c|c|c|c|c|c|}
\hline Function & & SSO [29] & NISSO [37] & MSSO [38] & OBSSO [39] & CLPSO [40] & JADE [41] & ISSO \\
\hline \multirow{4}{*}{ Sphere } & Min & $2.39 E-06$ & $5.70 E-20$ & $7.44 E-35$ & $4.57 E-28$ & $1.38 E-53$ & $8.22 E-45$ & $2.84 E-63$ \\
\hline & Mean & $6.88 E-06$ & $1.63 E-19$ & $1.40 E-34$ & $5.09 E-26$ & $1.70 E-52$ & $5.60 E-44$ & $7.05 E-62$ \\
\hline & Std & $7.06 E-07$ & $9.44 E-20$ & $8.21 E-35$ & $7.49 E-28$ & $4.41 E-53$ & $3.15 E-45$ & $1.11 E-62$ \\
\hline & At (s) & 7.47 & 5.86 & 7.05 & 5.34 & 4.14 & 4.94 & 3.68 \\
\hline \multirow{4}{*}{ SumSquare } & Min & $1.88 E-16$ & $4.66 E-32$ & $5.49 E-43$ & $8.73 E-35$ & $1.79 E-59$ & $5.14 E-52$ & $4.61 E-59$ \\
\hline & Mean & $4.30 E-14$ & $7.68 E-30$ & $4.87 E-42$ & $8.44 E-34$ & $8.60 E-57$ & $1.80 E-51$ & $3.87 E-58$ \\
\hline & Std & $5.79 E-15$ & $3.13 E-30$ & $9.40 E-43$ & $4.97 E-34$ & $9.63 E-58$ & $3.19 E-52$ & $7.53 E-59$ \\
\hline & At (s) & 7.41 & 5.79 & 7.14 & 5.61 & 4.05 & 5.01 & 3.74 \\
\hline \multirow{4}{*}{ Schwefel 2.21} & Min & $9.29 E-01$ & $5.11 E-01$ & $8.44 E-02$ & $5.94 E-01$ & $8.63 E-03$ & $2.05 E-02$ & $4.72 E-03$ \\
\hline & Mean & $3.52 E+01$ & $2.20 E+00$ & $2.01 E-01$ & $1.33 E+00$ & $2.24 E-02$ & $3.81 E-02$ & $7.38 E-03$ \\
\hline & Std & $3.03 E+00$ & $4.93 E-01$ & $4.62 E-02$ & $5.33 E-01$ & $9.82 E-03$ & $4.57 E-03$ & $9.46 E-04$ \\
\hline & At (s) & 7.69 & 5.83. & 6.93 & 5.44 & 4.10 & 4.86 & 3.79 \\
\hline \multirow{4}{*}{ Schwefel 2.22} & Min & $7.27 E-06$ & $5.60 E-09$ & $4.33 E-15$ & $2.61 E-12$ & $3.04 E-29$ & $7.83 E-24$ & $1.58 E-31$ \\
\hline & Mean & $5.69 E-04$ & $8.05 E-07$ & $4.20 E-14$ & $7.44 E-12$ & $3.47 E-27$ & $9.53 E-23$ & $4.84 E-31$ \\
\hline & Std & $2.56 E-04$ & $8.77 E-08$ & $6.73 E-15$ & $9.25 E-13$ & $6.89 E-28$ & $6.80 E-23$ & $9.15 E-32$ \\
\hline & At (s) & 7.51 & 5.90 & 7.02 & 5.29 & 4.22 & 4.57 & 3.70 \\
\hline \multirow{4}{*}{ Rosenbrock } & Min & $7.29 E+01$ & $3.60 E+01$ & $4.34 E-01$ & $2.05 E+00$ & $1.05 E-01$ & $5.60 E-01$ & $1.17 E-01$ \\
\hline & Mean & $1.14 E+02$ & $6.51 E+01$ & $1.39 E+00$ & $2.48 E+01$ & $1.88 E-01$ & $6.47 E-01$ & $1.20 E-01$ \\
\hline & Std & $1.58 E+01$ & $7.20 E+01$ & $1.11 E+01$ & $8.95 E+00$ & $1.03 E+00$ & $6.97 E-01$ & $2.93 E-01$ \\
\hline & At (s) & 7.60 & 5.94 & 7.15 & 5.48 & 4.27 & 4.95 & 3.91 \\
\hline \multirow{4}{*}{ Rastrigin } & Min & $7.23 E-02$ & $4.80 E-05$ & $3.09 E-09$ & $3.25 E-08$ & $6.64 E-14$ & $5.57 E-11$ & $2.31 E-14$ \\
\hline & Mean & $4.36 E-01$ & $6.49 E-04$ & $3.22 E-08$ & $8.20 E-08$ & $1.08 E-13$ & $4.51 E-10$ & $3.16 E-14$ \\
\hline & Std & $7.66 E-02$ & $9.17 E-05$ & $1.37 E-08$ & $9.79 E-09$ & $5.43 E-14$ & $9.03 E-11$ & $1.27 E-14$ \\
\hline & At (s) & 7.55 & 5.98 & 7.17 & 5.69 & 4.59 & 5.20 & 3.84 \\
\hline \multirow{4}{*}{ Ackley } & Min & $4.26 E-09$ & $7.15 E-10$ & $2.09 E-14$ & $8.32 E-10$ & $2.53 E-14$ & $3.28 E-14$ & $2.86 E-14$ \\
\hline & Mean & $2.30 E-06$ & $3.29 E-08$ & $7.83 E-14$ & $1.55 E-09$ & $6.14 E-14$ & $4.70 E-14$ & $3.30 E-14$ \\
\hline & Std & $4.61 E-07$ & $2.40 E-08$ & $9.76 E-15$ & $8.40 E-10$ & $1.25 E-13$ & $8.34 E-15$ & $6.87 E-15$ \\
\hline & At (s) & 7.63 & 6.03 & 7.09 & 5.58 & 4.19 & 4.97 & 3.77 \\
\hline \multirow{4}{*}{ Levy } & Min & $8.85 E-10$ & $3.19 E-16$ & $8.74 E-23$ & $4.89 E-23$ & $1.25 E-31$ & $7.82 E-27$ & $3.58 E-31$ \\
\hline & Mean & $3.41 E-08$ & $6.84 E-14$ & $1.48 E-22$ & $8.47 E-21$ & $5.63 E-31$ & $3.61 E-26$ & $4.25 E-31$ \\
\hline & Std & $1.37 E-08$ & $9.07 E-15$ & $4.61 E-23$ & $8.99 E-22$ & $3.01 E-31$ & $8.64 E-27$ & $2.53 E-31$ \\
\hline & At (s) & 7.56 & 6.15 & 7.01 & 5.45 & 4.16 & 5.03 & 3.62 \\
\hline
\end{tabular}

TABle 4: Comparisons of the results of 3-unit system with $P_{D} 850 \mathrm{MW}$.

\begin{tabular}{lcccc}
\hline Algorithm & Min & Mean & Max & At (s) \\
\hline SSO [29] & $\mathbf{8 2 3 4 . 0 7}$ & 8240.89 & 8244.15 & 0.97 \\
NISSO [37] & $\mathbf{8 2 3 4 . 0 7}$ & 8238.44 & 8242.04 & 0.91 \\
MSSO [38] & $\mathbf{8 2 3 4 . 0 7}$ & 8236.08 & 8240.57 & 0.72 \\
OBSSO [39] & $\mathbf{8 2 3 4 . 0 7}$ & 8235.96 & 8241.70 & 0.78 \\
CLPSO [40] & $\mathbf{8 2 3 4 . 0 7}$ & $\mathbf{8 2 3 4 . 0 7}$ & $\mathbf{8 2 3 4 . 0 7}$ & 0.29 \\
JADE [41] & $\mathbf{8 2 3 4 . 0 7}$ & $\mathbf{8 2 3 4 . 0 7}$ & $\mathbf{8 2 3 4 . 0 7}$ & 0.37 \\
CEP [1] & $\mathbf{8 2 3 4 . 0 7}$ & 8235.97 & 8241.83 & 8241.78 \\
FEP [1] & $\mathbf{8 2 3 4 . 0 7}$ & 8234.24 & 8241.80 & 8.45 \\
MFEP [1] & 8234.08 & 8234.71 & 8234.54 & $\mathbf{8 2 0}$ \\
IFEP [1] & $\mathbf{8 2 3 4 . 0 7}$ & 8234.16 & $\mathbf{8 2 3 4 . 0 7}$ & $\mathbf{0 . 1 2}$ \\
ISSO & $\mathbf{8 2 3 4 . 0 7}$ & $\mathbf{8 2 3 4 . 0 7}$ & & \\
\hline
\end{tabular}

provide an intuitive comparison of the 40-unit system ED problems.

Tables 4-6 show that the solutions obtained by the ISSO are almost better than those by any other methods in terms of minimum cost, mean cost, maximum cost, and average computing time regardless of the ED problem (i.e., 3-unit, 13-unit, and 40-unit). As for small-scale ED problem, almost all the compared algorithms can obtain the optimal solutions; nevertheless, only ISSO and/or CLPSO can achieve them as the scale of the units increases, which demonstrates a remarkable advantage of ISSO. Moreover, Figure 3 shows that the mean value is closer to each other among 30 trials compared with other algorithms. This finding adequately demonstrates that ISSO not only has high precision but also has strong stability. Figure 4 intuitively indicates that the convergence curve of ISSO is the steepest, which indicates that ISSO has an overwhelming advantage on convergence speed. Therefore, the improvement in standard SSO is 
Table 5: Comparisons of the results of 13-unit system with $P_{D} 1800 \mathrm{MW}$.

\begin{tabular}{|c|c|c|c|c|}
\hline Algorithm & Min & Mean & Max & At (s) \\
\hline $\begin{array}{l}\text { SSO [29] } \\
\end{array}$ & 18164.53 & 18288.43 & 18451.66 & 3.16 \\
\hline NISSO [37] & 18143.14 & 18232.08 & 18289.58 & 2.33 \\
\hline MSSO [38] & 18119.80 & 18186.50 & 18212.35 & 2.69 \\
\hline OBSSO [39] & 18136.42 & 18219.63 & 18294.75 & 2.01 \\
\hline CLPSO [40] & 17988.92 & 18095.04 & 18147.20 & 1.83 \\
\hline JADE [41] & 18073.61 & 18101.29 & 18193.68 & 1.96 \\
\hline CEP [1] & 18048.21 & 18190.32 & 18404.04 & 294.96 \\
\hline FEP [1] & 18018.00 & 18200.79 & 18453.82 & 168.11 \\
\hline MFEP [1] & 18028.09 & 18192.00 & 18416.89 & 317.12 \\
\hline IFEP [1] & 17994.07 & 18127.06 & 18267.42 & 157.43 \\
\hline ISSO & 17988.92 & 18064.37 & 18101.81 & 1.31 \\
\hline
\end{tabular}

Table 6: Comparisons of the results of 40-unit system with $P_{D} 10500 \mathrm{MW}$.

\begin{tabular}{lcccc}
\hline Algorithm & Min & Mean & Max \\
\hline SSO [29] & 123577.12 & 124812.78 & 126201.69 \\
NISSO [37] & 122896.60 & 124664.79 & 125884.04 & 5.26 \\
MSSO [38] & 122873.11 & 124100.01 & 124867.20 & 4.07 \\
OBSSO [39] & 123049.59 & 124471.71 & 124928.04 & 1.36 \\
CLPSO [40] & 122737.83 & 122800.96 & 122851.22 & 3.52 \\
JADE [41] & 122755.05 & 123356.12 & 124127.51 & 3.11 \\
CEP [1] & 123488.29 & 124793.48 & 126902.89 & 1956.93 \\
FEP [1] & 122679.71 & 124119.37 & 127245.59 & 1037.90 \\
MFEP [1] & 122647.57 & 123489.74 & 124356.47 & 2196.10 \\
IFEP [1] & 122624.35 & 123382.00 & 125740.63 & 1167.35 \\
ISSO & $\mathbf{1 2 2 5 1 9 . 2 4}$ & $\mathbf{1 2 2 5 7 5 . 2 4}$ & $\mathbf{1 2 2 7 3 1 . 0 7}$ \\
\hline
\end{tabular}

Table 7: Best dispatch obtained by ISSO for 3-unit system with $P_{D} 850 \mathrm{MW}$.

\begin{tabular}{lccc}
\hline Unit & 1 & 2 & 3 \\
\hline Output (MW) & 300.27 & 149.73 & 400.00 \\
Total cost $(\$)$ & & 8234.07 & \\
\hline
\end{tabular}

TABle 8: Best dispatch obtained by ISSO for 13-unit system with $P_{D} 1800 \mathrm{MW}$.

\begin{tabular}{|c|c|c|c|c|c|c|c|c|c|c|c|c|c|}
\hline Unit & 1 & 2 & 3 & 4 & 5 & 6 & 7 & 8 & 9 & 10 & 11 & 12 & 13 \\
\hline Output (MW) & 628.32 & 224.40 & 297.55 & 60.00 & 60.00 & 60.00 & 60.00 & 60.00 & 159.73 & 40.00 & 40.00 & 55.00 & 55.00 \\
\hline Total cost $(\$)$ & & & & & & & 17988.92 & & & & & & \\
\hline
\end{tabular}

TABle 9: Best dispatch obtained by ISSO for 40-unit system with $P_{D} 10500 \mathrm{MW}$.

\begin{tabular}{lcccccccccc}
\hline Unit & 1 & 2 & 3 & 4 & 5 & 6 & 7 & 8 & 9 \\
\hline Output (MW) & 113.81 & 110.94 & 119.93 & 179.71 & 96.91 & 140.00 & 300.00 & 300.00 & 284.64 & 130.00 \\
Unit & 11 & 12 & 13 & 14 & 15 & 16 & 17 & 18 & 19 & 20 \\
Output (MW) & 94.00 & 94.00 & 125.00 & 214.77 & 304.39 & 483.77 & 498.09 & 489.49 & 511.50 & 511.22 \\
Unit & 21 & 22 & 23 & 24 & 25 & 26 & 27 & 28 & 29 & 30 \\
Output (MW) & 523.27 & 546.12 & 549.56 & 549.47 & 549.97 & 549.76 & 10.21 & 10.00 & 10.03 & 88.18 \\
Unit & 31 & 32 & 33 & 34 & 35 & 36 & 37 & 38 & 39 & 40 \\
Output (MW) & 190.00 & 190.00 & 190.00 & 200.00 & 200.00 & 200.00 & 110.00 & 110.00 & 109.98 & 511.29 \\
\hline
\end{tabular}

Total cost (\$)

122519.24

effective, as presented in the following three aspects. First, subpopulation dynamically updating strategy is good for improving the diversity and quality of the subpopulation, and prematuration is prevented. Second, Gaussian mating radius ensures that the mating radius approaches a certain value with oscillation, which improves the efficiency of mating in a very clear way. Third, multimating operators, which refer to a large number of neighboring information of 


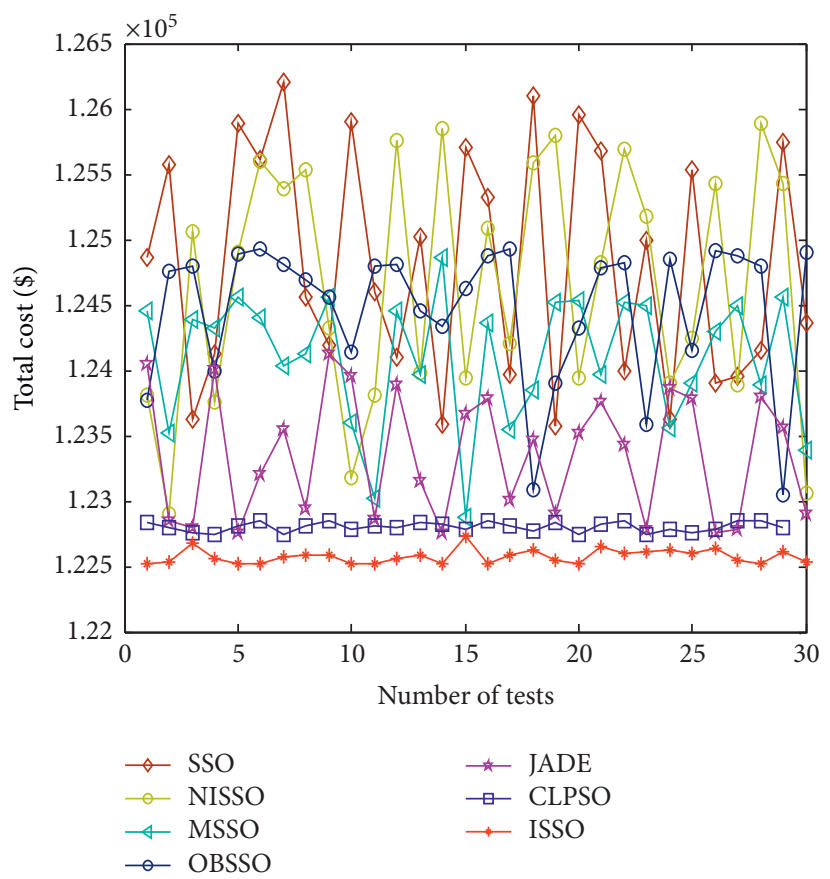

Figure 3: Mean of 30 tests of the 40 -unit system.

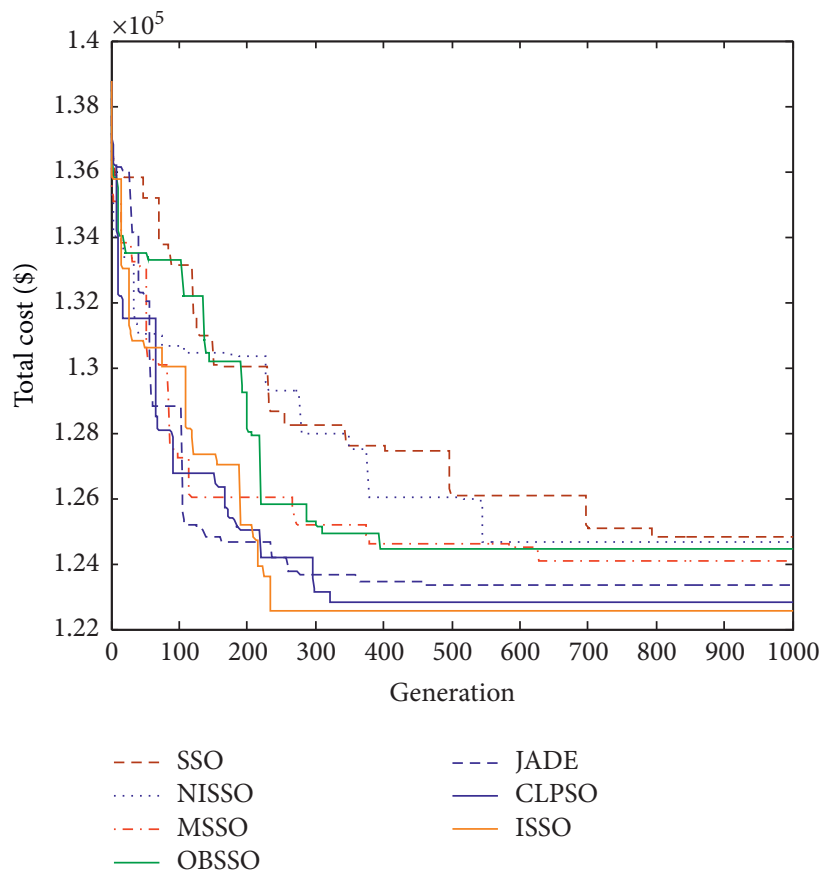

FIgURE 4: Convergence performance of the 40-unit system.

spiders, further increase the search depth and beneficial to ameliorate the accuracy of the solution to some extent. In summary, the abovementioned improvements on ISSO better balance exploration and exploitation.

\section{Conclusion}

A modified version of the conventional SSO, called ISSO, is presented in this paper to solve the ED problem with valve- 
point effects efficiently. Specifically, ISSO improves the subpopulation, mating radius, and mating operator of the SSO, remarkably enhances searching efficiency, and effectively avoids premature convergence. The comparison results of benchmark functions with some popular approaches demonstrate that high-quality solutions can be obtained by using the ISSO. Finally, three different scale ED problems with valve-point effects, which include 3-unit, 13-unit, and 40-unit, are solved with ISSO. The computational results show that ISSO has satisfactory solution precision and robustness, especially in large-scale problems. Moreover, the improvements in SSO are valid and reasonable. These improvements are also suitable for ED problems with valvepoint effects, which are characterized as nonsmooth, nonlinear, nonconvex, and nondifferentiable.

Considering the flexibility of the ED problem, future work will be addressed on dynamic ED problems, which are close to ED problems in real life. More importantly, ISSO can be effectively applied to ED problems in practical engineering.

\section{Data Availability}

The data used to support the findings of this study are supplied by the Henan Institute of Science and Technology under license and so cannot be made freely available. Requests for access to these data should be made to Wenqiang Yang at yangwqjsj@163.com.

\section{Conflicts of Interest}

The authors declare that they have no conflicts of interest.

\section{Acknowledgments}

This work was supported by the National Natural Science Foundation of China (nos. 61773156, 52077213, and 62003332), Scientific and Technological Project of Henan Province (nos. 202102110281 and 202102110282), Natural Science Foundation of Anhui Province (no. 2008085QE239), National Natural Science Foundation of Guangdong (nos. 2018A030310671 and 2016A030313177), and Outstanding Young Researcher Innovation Fund of Shenzhen Institute of Advanced Technology, Chinese Academy of Sciences (no. 201822).

\section{References}

[1] N. Sinha, R. Chakrabarti, and P. K. Chattopadhyay, "Evolutionary programming techniques for economic load dispatch," IEEE Transactions on Evolutionary Computation, vol. 7, no. 1, pp. 83-94, 2003.

[2] S. Ghaffari, G. Aghajani, A. Noruzi, and H. Hedayati Mehr, "Optimal economic load dispatch based on wind energy and risk constrains through an intelligent algorithm," Complexity, vol. 21, no. 2, pp. 494-506, 2016.

[3] G. Hou, L. Gong, Z. Yang, and J. Zhang, "Multi-objective economic model predictive control for gas turbine system based on quantum simultaneous whale optimization algorithm," Energy Conversion and Management, vol. 207, Article ID 112498, 2020.
[4] Z. Yang, K. Liu, J. Fan, Y. Guo, Q. Niu, and J. Zhang, "A novel binary/real-valued pigeon inspired optimization for economic/environment unit commitment with renewables and plug-in vehicles," Science China Information Science, vol. 62, Article ID 070213, 2019.

[5] P. Oliveira, S. Mckee, and C. Coles, "Lagrangian relaxation and its application to the unit-commitment-economic-dispatch problem," IMA Journal of Management Mathematics, vol. 4, no. 3, pp. 261-272, 1992.

[6] R. A. Jabr, A. H. Coonick, and B. J. Cory, "A homogeneous linear programming algorithm for the security constrained economic dispatch problem," IEEE Transactions on Power Systems, vol. 15, no. 3, pp. 930-936, 2000.

[7] C. L. Chen and S. C. Wang, "Branch-and-bound scheduling for thermal generating units," IEEE Transactions on Energy Conversion, vol. 8, no. 2, pp. 184-189, 1993.

[8] J. Y. Fan and L. Zhang, "Real-time economic dispatch with line flow and emission constraints using quadratic programming," IEEE Transactions on Power Systems, vol. 13, no. 2, pp. 320-325, 1998.

[9] Q. Chen, H. Shi, and M. Sun, "Echo state network-based backstepping adaptive iterative learning control for strictfeedback systems: an error-tracking approach," IEEE Transactions on Cybernetics, vol. 50, no. 7, pp. 3009-3022, 2020.

[10] Q. Chen, X. Yu, M. Sun, C. Wu, and Z. Fu, “Adaptive repetitive learning control of PMSM servo systems with bounded nonparametric uncertainties: theory and experiments," IEEE Transactions on Industrial Electronics, p. 1, 2020.

[11] S. Wang, L. Tao, Q. Chen, J. Na, and X. Ren, "USDE-based sliding mode control for servo mechanisms with unknown system dynamics," IEEE/ASME Transactions on Mechatronics, vol. 25, no. 2, pp. 1056-1066, 2020.

[12] J. Na, Y. Li, Y. Huang, G. Gao, and Q. Chen, "Output feedback control of uncertain hydraulic servo systems," IEEE Transactions on Industrial Electronics, vol. 67, no. 1, pp. 490-500, 2020.

[13] M. Nemati, M. Braun, and S. Tenbohlen, "Optimization of unit commitment and economic dispatch in microgrids based on genetic algorithm and mixed integer linear programming," Applied Energy, vol. 210, pp. 944-963, 2017.

[14] D. C. Walters and G. B. Sheble, "Genetic algorithm solution of economic dispatch with valve point loading," IEEE Transactions on Power Systems, vol. 8, no. 3, pp. 1325-1332, 1993.

[15] T. A. A. Victoire and A. E. Jeyakumar, "Hybrid PSO-SQP for economic dispatch with valve-point effect," Electric Power Systems Research, vol. 71, no. 1, pp. 51-59, 2004.

[16] Q. Niu, Z. Zhou, H.-Y. Zhang, and J. Deng, “An improved quantum-behaved particle swarm optimization method for economic dispatch problems with multiple fuel options and valve-points effects," Energies, vol. 5, no. 9, pp. 3655-3673, 2012.

[17] N. Yousefi, "Solving nonconvex economic load dispatch problem using particle swarm optimization with time varying acceleration coefficients," Complexity, vol. 21, no. 6, pp. 299-308, 2016.

[18] O. Abedinia, A. Ghasemi, and N. Ojaroudi, "Improved time varying inertia weight PSO for solved economic load dispatch with subsidies and wind power effects," Complexity, vol. 21, no. 4, pp. 40-49, 2016.

[19] Z. Yang, K. Li, Y. Guo et al., "A binary symmetric based hybrid meta-heuristic method for solving mixed integer unit commitment problem integrating with significant plug-in electric vehicles," Energy, vol. 170, pp. 889-905, 2019.

[20] D. Singh and J. S. Dhillon, "Ameliorated grey wolf optimization for economic load dispatch problem," Energy, vol. 169, pp. 398-419, 2019. 
[21] I. Ziane, F. Benhamida, and A. Graa, "Simulated annealing algorithm for combined economic and emission power dispatch using max/max price penalty factor," Neural Computing \& Applications, vol. 28, no. 1, pp. 197-205, 2017.

[22] C. Tsai, D. Thikien, and T. Pan, "Parallel bat algorithm applied to the economic load dispatch problem," Journal of Internet Technology, vol. 17, no. 4, pp. 761-769, 2016.

[23] H. Ma, Z. Yang, P. You, and M. Fei, "Multi-objective biogeography-based optimization for dynamic economic emission load dispatch considering plug-in electric vehicles charging," Energy, vol. 135, pp. 101-111, 2017.

[24] N. Noman and H. Iba, "Differential evolution for economic load dispatch problems," Electric Power Systems Research, vol. 1, no. 78, pp. 1322-1331, 2008.

[25] M. Nazari-Heris, M. Mehdinejad, B. Mohammadi-Ivatloo, and G. Babamalek-Gharehpetian, "Combined heat and power economic dispatch problem solution by implementation of whale optimization method," Neural Computing and Applications, vol. 31, no. 2, pp. 421-436, 2019.

[26] Z. Yang, K. Li, Q. Niu, Y. Xue, and A. Foley, “A self-learning TLBO based dynamic economic/environmental dispatch considering multiple plug-in electric vehicle loads," Journal of Modern Power Systems and Clean Energy, vol. 2, no. 4, pp. 298-307, 2014.

[27] E. Afzalan and M. Joorabian, "An improved cuckoo search algorithm for power economic load dispatch," International Transactions on Electrical Energy Systems, vol. 25, no. 6, pp. 958-975, 2015.

[28] T. T. Nguyen, T. T. Nguyen, and D. N. Vo, “An effective cuckoo search algorithm for large-scale combined heat and power economic dispatch problem," Neural Computing and Applications, vol. 30, no. 11, pp. 3545-3564, 2017.

[29] E. Cuevas, M. Cienfuegos, D. Zaldívar, and M. Pérez-Cisneros, "A swarm optimization algorithm inspired in the behavior of the social-spider," Expert Systems with Applications, vol. 40, no. 16, pp. 6374-6384, 2013.

[30] C. Erik and C. Miguel, "A new antilogarithm inspired in the behavior of social-spider for constrainedoptimization," Expert System with Applications, vol. 41, no. 1, pp. 412-425, 2014.

[31] U. P. Shukla and S. J. Nanda, "Dynamic clustering with binary social spider algorithm for streaming dataset," Soft Computing, vol. 23, no. 21, pp. 10717-10737, 2019.

[32] J. J. Q. Yu and V. O. K. Li, "A social spider algorithm for solving the non-convex economic load dispatch problem," Neurocomputing, vol. 171, pp. 955-965, 2015.

[33] J. Qiu, J. Xie, and F. Cheng, "A hybrid social spider optimization algorithm with differential evolution for global optimization," Journal of Universal Computer Science, vol. 23, no. 7, pp. 619-635, 2017.

[34] Y. Zhang, X. Qian, J. Wang, and M. Gendeel, "Fuzzy rulebased classification system using multi-population quantum evolutionary algorithm with contradictory rule reconstruction," Applied Intelligence, vol. 49, no. 11, pp. 4007-4021, 2019.

[35] A. Godio, "Multi population genetic algorithm to estimate snow properties from GPR data," Journal of Applied Geophysics, vol. 131, pp. 133-144, 2016.

[36] M. Z. Ali, N. H. Awad, and P. N. Suganthan, "Multi-population differential evolution with balanced ensemble of mutation strategies for large-scale global optimization," Applied Soft Computing, vol. 33, pp. 304-327, 2015.

[37] T. N. Thang, "A high performance social spider optimization algorithm for optimalpower flow solution with single objective optimization," Energy, vol. 171, no. 15, pp. 218-240, 2019.
[38] C. E. Klein, E. H. V. Segundo, V. C. Mariani, and L. D. S. Coelho, "Modified social-spider optimization algorithm applied to electromagnetic optimization," IEEE Transactions on Magnetics, vol. 52, no. 3, pp. 1-4, 2016.

[39] R. A. Ibrahim, M. A. Elaziz, D. Oliva, E. Cuevas, and S. Lu, "An opposition-based social spider optimization for feature selection," Soft Computing, vol. 23, no. 24, pp. 13547-13567, 2019.

[40] J. J. Liang, A. K. Qin, P. N. Suganthan, and S. Baskar, "Comprehensive learning particle swarm optimizer for global optimization of multimodal functions," IEEE Transactions on Evolutionary Computation, vol. 10, no. 3, pp. 281-295, 2006.

[41] J. Q. Zhang and A. C. Sanderson, "JADE: Adaptive differential evolution with optional external archive," IEEE Transactionson Evolutionary Computation, vol. 13, no. 5, pp. 945958, 2009.

[42] W. Gao, F. T. S. Chan, L. Huang, and S. Liu, "Bare bones artificial bee colony algorithm with parameter adaptation and fitness-based neighborhood," Information Sciences, vol. 316, pp. 180-200, 2015. 